\title{
Milieux professionnels et FASP médicale : de l'autre côté du miroir
}

Jean-Pierre Charpy

\section{OpenEdition}

\section{Journals}

Édition électronique

URL : http://journals.openedition.org/asp/866

DOI : $10.4000 /$ asp.866

ISBN : 978-2-8218-0398-5

ISSN : 2108-6354

Éditeur

Groupe d'étude et de recherche en anglais de spécialité

Édition imprimée

Date de publication : 1 décembre 2004

Pagination : 43-59

ISSN : 1246-8185

\section{Référence électronique}

Jean-Pierre Charpy, « Milieux professionnels et FASP médicale : de l'autre côté du miroir », ASp [En ligne], 45-46 | 2004, mis en ligne le 02 mars 2010, consulté le 01 mai 2019. URL : http:// journals.openedition.org/asp/866; DOI : 10.4000/asp.866

Ce document a été généré automatiquement le 1 mai 2019.

Tous droits réservés 


\title{
Milieux professionnels et FASP médicale : de l'autre côté du miroir
}

\author{
Jean-Pierre Charpy
}

\section{Introduction}

1 Depuis les années 1970, des centaines de romans à suspense à dominante médicale écrits par des professionnels de la santé devenus écrivains ont été publiés aux États-Unis. Leur succès populaire a rapidement dépassé les frontières de l'Amérique du Nord et, aujourd'hui, des auteurs comme R.Cook ou M.Palmer jouissent d'une réputation internationale.

2 Ces medical thrillers ont comme dénominateur commun la représentation du domaine professionnel de la médecine perceptible, à des degrés divers, dans la trame narrative et le tissu linguistique spécialisé qui les caractérisent. Cette prégnance de la réalité médicale est telle que les éditeurs et les auteurs aiment à dire que ces romans reflètent les pratiques et le discours des milieux professionnels de la santé.

3 Dans cette étude, nous proposons d'aller au-delà des constatations de surface pour porter un regard approfondi sur les relations entre la fiction à substrat professionnel (FASP) médicale et l'environnement professionnel. Nous tentons de montrer, en nous référant à divers travaux de recherche sur la FASP, comment un genre littéraire existant, à savoir celui du roman à suspense, a été phagocyté par un substrat professionnel à dominante ou à forte influence médicale.

4 La première étape de notre travail consiste à placer le roman à suspense à dominante médicale dans une perspective historique et à définir la typologie de ce sous-groupe générique. L'étape suivante de notre analyse porte sur le repérage systématique, dans le paratexte éditorial, graphique et auctorial des romans médicaux, des principales caractéristiques formelles externes permettant de confirmer l'appellation de sous-groupe générique à dominante professionnelle. Les caractéristiques formelles internes des ouvrages les plus connus sont également répertoriées dans une troisième partie. Elles 
mettent en évidence - au-delà des stéréotypes - l'intégration en profondeur de certaines pratiques et d'éléments du discours de la communauté médicale dans la narration. La quatrième étape vise à affiner notre analyse initiale des milieux professionnels et à clarifier le statut parfois ambigu des auteurs de medical thrillers en passant de l'autre côté du miroir. Nous y présentons une synthèse des réponses à un questionnaire ciblé soumis à quatre écrivains représentatifs : R. Cook, M. Palmer, L.R. Robinson et T. Gerritsen.

\section{Fiction à substrat professionnel et FASP médicale}

\subsection{FASP et substrat professionnel}

Si le roman à suspense ou à énigme, communément appelé thriller, a une histoire déjà ancienne ${ }^{1}$, le genre de la fiction à substrat professionnel a été défini récemment :

Nous postulons que cet ensemble d'ouvrages de fiction présente suffisamment de caractéristiques communes pour justifier une appellation générique, et que la nature de ces caractéristiques peut être résumée par l'appellation de «substrat professionnel ", ce qui nous conduira donc à parler de fiction à substrat professionnel, ou FASP. (Petit 1999 : 57)

Les formes spécialisées de ce genre sont le legal thriller, le military thriller, le scientific thriller, le technothriller, le political thriller, le forensic thriller et le medical thriller. Il s'agit en fait d'un agglomérat de romans caractérisés par l'utilisation conjointe d'un discours spécialisé à dominante professionnelle et d'une intrigue marquée par le recours au suspense.

7 Cet extrait du paratexte éditorial de The Hunt for Red October de T. Clancy souligne l'osmose entre substrat professionnel authentique et frissons : «A fine thriller [...] flawless authenticity, frighteningly genuine » (Wall Street Journal).

G. Diaz-Santos (2000) revient sur les caractéristiques majeures de certains romans appartenant au domaine de recherche de la FASP, tel que M. Petit l'a défini :

During the last few years, doctors, biologists or physicists-turned-writers have raised science and high-tech topics to the level of hot literary commodities. Medical plots, deadly viruses, code-breaking, genetic manipulation, computer security, or virtual information environments are now themes which constitute creditable examples of how fast-paced action can be combined with scientific discussion and yield well-documented fiction works which entrance and educate a wide readership at the same time. $(2000: 222)$

9 Tous ces éléments convergents montrent que le roman à suspense à dominante médicale fait partie intégrante d'un genre populaire destiné au profane, mais qui s'appuie sur l'expérience et le discours des milieux professionnels. Dans son article fondateur, M. Petit fait la remarque suivante au sujet de ces romans spécialisés :

Ils ont en commun de reposer sur un certain type de rapport avec ce milieu, le milieu lui-même n'étant en quelque sorte qu'une variable. C'est l'existence de ce rapport qui nous paraît définitoire du genre. (1999: 62)

10 Il convient désormais de définir les traits distinctifs de ce sous-groupe générique de la FASP, que nous nommerons désormais FASP médicale. 


\subsection{La FASP médicale : historique d'un sous-groupe générique}

11 Comme le laisse entrevoir le paratexte éditorial de Blood Run de L.R. Robinson, le roman à suspense à dominante médicale est le résultat de la fusion entre la matière médicale brute et les feux d'une intrigue marquée par de fréquents rebondissements : "A thrilling blend of cold medical facts and heated emotions » (The New York Times Book Review).

12 F.G. Slaughter et M. Crichton sont les principaux inspirateurs de ce type de fiction médicale fondée sur l'expérience professionnelle des auteurs. F.G. Slaughter est souvent décrit comme le maître du roman médical car il a su mettre son expérience de chirurgien au service de l'écriture, s'attachant à dépeindre sans concession la communauté médicale qu'il connaissait de l'intérieur, comme dans cet extrait de Doctors at Risk :

When the scope of what the AMA called the 'Impaired Physician' problem was first realized five years ago, it was hoped that doctors whose abilities - their whole lives, in fact - are crippled by alcoholism or drug addiction would seek aid of their own accord, John Carr continued. Unfortunately, that rarely occurs, and they usually don't come to the attention of the committees until the drinking or drug problem is severe enough to cause their licenses to be in danger of being revoked by the Medical Licensing Board. (1983 : 188-189)

13 Les romans de F.G. Slaughter présentent un intérêt sociologique et linguistique indéniable, mais ils comportent peu d'éléments de suspense ${ }^{2}$. À ce titre, cet auteur ne peut pas être considéré comme le père fondateur de la FASP médicale, d'autant plus qu'il a également écrit de nombreux livres d'inspiration religieuse ou historique.

Quant à M. Crichton, il a été un des premiers à utiliser le substrat de la langue et du discours professionnel de façon homogène dans des romans à suspense - A Case of Need (1968), The Terminal Man (1972) - ou dans l'adaptation cinématographique du premier roman médical de R. Cook, Coma (Turner Entertainment Co, 1978). Toutefois, il a délaissé la pratique médicale à l'issue de ses études de médecine pour se consacrer à l'écriture de technothrillers, autre sous-groupe générique de la FASP dont il est devenu la figure emblématique.

richton a inspiré les auteurs de la FASP médicale, les véritables fondateurs du roman à suspense à dominante médicale sont R. Cook et M. Palmer. À la fin des années soixante-dix, ces deux médecins devenus écrivains ont commencé à produire et à populariser ce qu'il est convenu d'appeler aujourd'hui le medical thriller.

une lettre aux lecteurs placée en exergue de l'édition Signet de Coma, R. Cook s'attribue clairement la paternité du sous-groupe générique dont nous allons étudier les principales caractéristiques :

On a lighter note, Coma essentially created a popular new genre: the medical/ biotech mystery thriller. I am pleased that the book's reception vindicated my belief that stories of social significance involving medicine and medical research could be as entertaining and engaging as those dealing with detectives, spies, political intrigue, and traditional whodunits. (1978: ii)

17 Il est intéressant de noter que, dans le paratexte éditorial, R. Cook est systématiquement présenté comme un professionnel de la médecine qui en est venu à écrire des romans spécialisés : «Leave it to doctor-turned-novelist Robin Cook to scare us all to death 》 (Los Angeles Times, au sujet de Invasion). immédiat de R. Cook, est tout aussi étroitement lié au milieu professionnel dont il est issu 
(et auquel il appartient toujours ${ }^{3}$ ). Dans le paratexte éditorial de The Patient, l'expérience professionnelle de l'auteur est mise en exergue : «A top-notch thriller [...] filled with bits and pieces that only someone who has lived his or her life in the medical profession can convey " (The Colony Times).

Avec la publication de dizaines de romans à suspense à dominante médicale, R. Cook et M. Palmer ont fortement contribué à la popularité de ce genre reposant sur l'expérience médicale des auteurs. Ils ont permis au medical thriller de prendre corps et, en ce sens, nous pensons qu'ils ont fait école. Leur succès a incité d'autres professionnels de la santé à quitter, provisoirement ou définitivement, la pratique de la médecine pour se consacrer à l'écriture. L.R. Robinson, qui travaille comme auxiliaire médicale volontaire, a fait quelques infidélités au service des urgences d'un hôpital new-yorkais pour réinvestir son expérience personnelle dans l'intrigue médicale (Blood Run, Unnatural Causes).

T. Gerritsen a abandonné la pratique de la médecine pour pouvoir élever ses enfants et produire, dans un premier temps, d'innocentes histoires romanesques. Depuis 1996, elle écrit des medical thrillers dont les plus connus sont Harvest, The Surgeon et The Apprentice. Dans ces romans, rédigés avec une précision chirurgicale, l'innocence, à tout jamais bannie de l'environnement médical, fait place aux plus noirs desseins et aux plus sombres peurs ${ }^{4}$.

21 Tous ces auteurs - pour ne citer que les plus représentatifs d'entre eux - ont fait du roman à suspense à dominante médicale un sous-groupe générique reconnu par de nombreux éditeurs et le grand public, mais aussi par les médecins et les praticiens hospitaliers eux-mêmes.

Toutefois, le statut de ces auteurs reste ambigu. Sont-ils devenus des écrivains à part entière s'appuyant uniquement sur leur expérience professionnelle passée ou ont-ils gardé un contact suffisant avec leur milieu professionnel initial pour pouvoir prétendre être des médecins qui, accessoirement, écrivent des romans spécialisés? C'est une question que nous leur avons soumise et dont les réponses seront fournies dans la quatrième partie de cet article.

Une autre question se pose dont il convient de vérifier la pertinence. À l'exception de quelques auteurs (L.S. Goldberg, le médecin irlandais P. Carson...) qui ont choisi d'autres régions des États-Unis comme toile de fond, la plupart des auteurs à succès ayant contribué à l'essor du medical thriller situent l'action de leurs romans en NouvelleAngleterre. Bon nombre d'entre eux (R. Cook, M. Palmer, L.R. Robinson...) ont, ou ont eu, un ancrage professionnel dans la Nouvelle-Angleterre et entretiennent des liens étroits avec le milieu médical de la ville de Boston.

Dans Flashback, M. Palmer, qui, à l'instar de R.Cook, a fait son internat dans deux hôpitaux de cette ville, insiste sur le rôle moteur joué par cette région au sein de la communauté médicale américaine :

The company had set its sights on the prestige that involvement with established medical schools would bring, and there were ten of the world's most respected institutions in New England alone. (1995: 213)

En raison de ce faisceau de convergences et du fait que certains auteurs (T. Gerritsen et M. Palmer) co-animent des ateliers d'écriture spécialisés ${ }^{5}$, nous avons émis, dans un article récent ${ }^{6}$, l'hypothèse de l'existence d'une "École de la Nouvelle-Angleterre " susceptible d'avoir posé les fondements théoriques du roman à suspense à dominante médicale. 
26 Nous avons soumis cette hypothèse de travail à quatre auteurs de la Nouvelle-Angleterre, R. Cook, M. Palmer, L.R. Robinson et T. Gerritsen. Leurs réactions seront présentées dans la quatrième partie, lorsque nous aurons défini les caractéristiques formelles externes et internes de ce sous-groupe générique.

\section{Typologie du roman à suspense à dominante médicale : caractéristiques formelles externes}

Le problème posé est celui du degré d'intégration des pratiques et du discours des professions de la santé dans les romans à suspense à dominante médicale.

Les maisons d'édition spécialisées mettent en valeur les caractéristiques professionnelles de ce type de fiction au travers d'éléments paratextuels susceptibles de capter et de retenir l'attention du lecteur. En surface, un simple survol des premières et des quatrièmes de couverture de ces romans permet de repérer des éléments textuels et visuels emblématiques de la communauté médicale. Il convient toutefois d'aller au-delà de cette première impression pour cerner les caractéristiques génériques externes de la FASP médicale en étudiant systématiquement ce qui gravite autour du texte.

G. Genette (1987) a défini les composantes essentielles du paratexte, définitoires du cadrage générique. Son approche théorique, visant à faire ressortir les caractéristiques formelles externes du paratexte, a été relayée par P. Lane :

Le paratexte désigne un ensemble de productions discursives qui accompagnent le texte ou le livre, comme la couverture, la jaquette, le prière d'insérer ou la publicité, le catalogue ou la presse d'édition. (1992: 9)

Nous traiterons $\mathrm{du}$ paratexte éditorial, du paratexte graphique, qui regroupe les composantes visuelles du paratexte éditorial, et du paratexte auctorial. Toutefois, dans un souci de simplification, nous ne prendrons pas en compte les nuances établies par G. Genette entre le péritexte, qui recouvre la matérialité du livre, et l'épitexte, qui concerne les événements éditoriaux et médiatiques situés à la périphérie du texte.

\subsection{Reflets de l'expérience professionnelle et paratexte éditorial}

31 Comme M. Petit l'a souligné dans son article séminal sur la FASP (1999), les commentaires éditoriaux ou les extraits de presse reproduits en première, en quatrième de couverture ou en pages intérieures répondent avant tout à des stratégies commerciales. L'accroche publicitaire la plus fréquemment mise en exergue sur la couverture est sans doute celle de «National bestseller», de «New York Times bestseller» ou de «International bestselling author ».

32 Le paratexte éditorial permet également de donner un cadre chronologique, voire historique aux romans de la FASP médicale. Ainsi, le premier roman à suspense à dominante médicale écrit par R. Cook est-il présenté comme une œuvre pour ainsi dire classique : «The classic medical thriller » (note éditoriale au sujet de Coma).

Pour établir la réputation d'un auteur, il est parfois fait allusion à un roman antérieur qui sert désormais de référence. T. Gerritsen est décrite de la manière suivante : «New York Times bestselling author of Harvest » (couverture de Life Support). 

Harvest) $1987: 31)$

Dans les rééditions, il est souvent fait allusion à l'ouvrage le plus récent de l'auteur. Cette stratégie est systématique chez Bantam Books : «Michael Palmer. Author of The Patient » (couverture de Flashback).

Si les formes canoniques medical thriller ou novel of medical suspense ne sont pas toujours présentes dans le paratexte éditorial, elles font l'objet d'une reformulation qui met en avant les éléments de suspense et / ou l'expérience professionnelle de l'auteur :

Palmer [brings] his fascinating ER procedural knowledge to a fast-paced [...] narrative. (San Francisco Chronicle au sujet de Critical Judgment)

Only a superb storyteller with a medical background such as Gerritsen's could have written such an eye-opening, palpitating thriller. (Maine Sunday Telegram au sujet de

Ces éléments du paratexte éditorial soulignent l'appartenance (passée ou présente) des auteurs à la communauté médicale, leur connaissance du champ professionnel concerné et la prégnance du suspense dans la narration. Ils constituent un faisceau de repères clairs visant à donner une crédibilité professionnelle à l'œuvre de fiction. Parfois, le paratexte éditorial se veut redondant et métaphorique afin de renforcer le réalisme médical du roman : "Murder is epidemic in this scalpel-sharp medical thriller » (au sujet de A Deadly Practice de L.S. Goldberg).

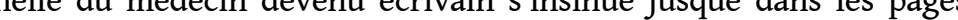
intérieures. L'éditeur en profite pour promouvoir d'autres romans, de même nature, écrits par l'auteur :

Spellbinding ... a chillingly sinister novel made all the more frightening by

[Palmer's] medical authority. (The Denver Post, au sujet de Extreme Measures, dans le paratexte éditorial de Side Effects)

Ce souci de validation professionnelle est également présent dans les éléments graphiques du paratexte auctorial.

\subsection{Images du milieu professionnel et paratexte graphique}

9 En observant la première et la quatrième de couverture de la plupart des medical thrillers, le regard du lecteur potentiel ne peut qu'être attiré par un titre choc à dominante médicale ${ }^{7}$ et des éléments visuels frappants évoquant les milieux professionnels de la santé (caducée, seringue, brancard, tracé sinusal, pipette, cupule...). Ces signes extérieurs, souvent redondants, de richesse médicale présents dans le paratexte graphique représentent un vecteur promotionnel essentiel.

Comme nous l'avons déjà indiqué (Charpy 2001), en faisant référence à A. Sauvageot, la puissance évocatrice de l'image crédibilise le propos professionnel attendu :

L'image, au fond, non seulement fascine, mais elle convainc. En effet, l'image et son contenu symbolique, en recréant une situation réelle qui tend vers la perfection, mettent le spectateur hors de toute possibilité d'intervention active. (Sauvageot

Des éléments visuels récurrents (caducée, tracé sinusal) sont présents sur la couverture de Life Support. Le caducée est reproduit en tête des vingt-et-un chapitres et de la page des références bibliographiques du roman de T.Gerritsen, donnant ainsi au récit une estampille professionnelle visible tout au long du roman. Il s'agit d'ailleurs du symbole graphique le plus fréquemment utilisé en couverture. On le retrouve, représenté de 
manière réaliste ou stylisée, dans A Case of Need, Doctors at Risk, Life Support et A Deadly Practice.

La seringue symbolise également le milieu médical sur les couvertures de Doctors at Risk, The Sisterhood et Natural Causes. D'autres stratégies éditoriales mettent en avant des accroches visuelles plus racoleuses; sinistres cadavres sur un brancard (Side Effects, Fatal) ou dans une morgue (Vector), jeune femme nue, potentielle victime d'un tueur en série ( The Surgeon, The Apprentice).

Le choix des couleurs est également important. La couleur rouge connote à la fois le sang, emblématique de l'accident et de la salle d'opération, et le danger ou l'urgence, tous deux moteurs du suspense dans la narration. Les jaquettes de Flashback, Chromosome 6 et Final Duty sont rouge écarlate.

Les titres de Fever, Vital Signs, Natural Causes, Blood Run, Shock et Fatal sont écrits en gros caractères de couleur rouge. La main ensanglantée qui ponctue le titre Blood Run, vient même renforcer cette impression d'urgence et de danger.

Afin de capter l'attention du lecteur ou de le fidéliser, une autre accroche visuelle est utilisée dans le paratexte graphique; il s'agit de l'image de l'auteur. La photographie de R. Cook en tenue de médecin est pratiquement toujours présente en quatrième de couverture. L'objectif est de donner une caution médicale à ses romans à succès, même si son expérience professionnelle est désormais lointaine. Dans ses thrillers les plus récents ( Vector, Shock), cette photographie occupe la totalité de la quatrième de couverture.

M. Palmer, T. Gerritsen et L.R. Robinson sont généralement relégués en troisième de couverture, où leur photographie est accompagnée d'une courte bibliographie. Il est intéressant de noter que dans Blood Run, l'auteur est photographiée devant une ambulance des services d'urgence, ce qui met en avant le contexte professionnel.

En revanche, en quatrième de couverture de Doctors at Risk, F.G. Slaughter dédicace un de ses romans devant les rayons d'une bibliothèque. Cette image de l'écrivain bien établi estompe quelque peu celle du médecin/chirurgien.

\section{3. Échos du milieu professionnel et paratexte auctorial}

48 À côté des illustrations graphiques du paratexte éditorial, de nombreuses interventions auctoriales viennent ancrer l'œuvre de fiction dans la réalité du milieu médical. En effet, le paratexte auctorial fait fréquemment référence à l'expérience professionnelle d'experts ou de spécialistes du monde médical qui apportent une caution scientifique à l'œuvre de fiction.

Dans la partie Acknowledgements, les écrivains consacrent souvent une page complète aux remerciements dûs aux divers professionnels qui les ont conseillés et guidés. R. Cook et P. Carson ne remercient pas moins de six chirurgiens ou médecins dans le paratexte auctorial de Vector et de Final Duty.

50 Dans le paratexte auctorial de Natural Causes, M. Palmer insiste sur les conseils précieux fournis par des spécialistes de son domaine professionnel, mais aussi par des juristes :

Dr. Rick Abisla of Falmouth, Massachusetts, and Dr. Dolores Emspak of Swampscott, Massachusetts, gave me invaluable technical advice in their specialty. Attorneys Marcia Divoll and Joanne Colombani Smith of Boston, and Ms. Ginni Ward did the same for me in their areas of the law. (1999: vii) 
51 citation précédant généralement le prologue ou le premier chapitre. L.S. Goldberg cite King Lear en exergue de A Deadly Practice: «Kill thy physician and thy fee bestow upon the foul disease » (1994 : vii). de A Case of Need. Il conclut son roman par des notes personnelles, à savoir sept annexes qui lui permettent d'exprimer son point de vue sur des sujets aussi variés que l'éthique médicale, l'avortement ou la terminologie médicale.

D'autres romanciers éprouvent le besoin de clarifier une terminologie parfois absconse pour le profane en incluant un glossaire à la fin du roman. C'est le cas de Vector et Chromosome 6 de R. Cook où l'auteur fournit de précieuses indications dans le domaine de la génétique et des transplantations.

Afin d'aider le lecteur à surmonter certaines difficultés techniques ou conceptuelles liées au substrat professionnel présent, certains auteurs, comme M. Palmer dans la partie Author's note de Fatal, proposent les coordonnées d'organismes spécialisés :

As a medical fiction author, it is often difficult for me to fully discuss complex medical issues in the course of a novel. Fatal is no exception. For those readers who would like to do their own investigation into these subjects, the following sources of information may be helpful. (2003: 510)

Enfin, une bibliographie détaillée proposée par l'auteur permet au lecteur d'approfondir ses connaissances dans le champ professionnel ciblé. Pas moins de cinquante et un ouvrages sont référencés à la fin de The Terminal Man de M. Crichton. Life Support de T. Gerritsen comporte une bibliographie comportant treize références.

Les divers éléments du paratexte présentés ci-dessus concourent tous à mettre en relief la prégnance de la réalité professionnelle dans les romans à suspense à dominante médicale.

\section{Typologie du roman à suspense à dominante médicale : caractéristiques formelles internes}

\subsection{Un tissu narratif à dominante professionnelle}

Après avoir passé en revue les principales caractéristiques formelles externes du roman à suspense à dominante médicale, nous allons en recenser les caractéristiques formelles internes en relation avec le milieu professionnel de la médecine dont les auteurs sont issus.

Les medical thrillers sont inscrits dans un cadre formel clairement identifiable qui répond à des critères de stabilité et de reconnaissance propres au sous-groupe générique de la FASP médicale : «Genres themselves are classes of communicative events which typically possess features of stability, name recognition and so on ... » (Swales 1990 : 9).

Un genre littéraire possède une unité formelle et conceptuelle qui assure à la narration une cohérence particulière. Dans le cas de la FASP médicale, le terme de sous-groupe générique paraît adéquat car les principaux traits du roman à suspense (intrigue complexe, recours systématique aux émotions fortes, poursuites échevelées, dénonciation de la corruption ou de la collusion, enquêtes policières...) s'entrecroisent avec les éléments constitutifs de l'environnement professionnel médical. 
60 L'interpénétration des thèmes majeurs du roman à suspense et du substrat professionnel propre à la communauté médicale est perceptible dans Vital Signs et Shock (corruption et manipulations génétiques), Fever, Final Duty, Critical Judgment et Miracle Cure (corruption, industrie pharmaceutique et problèmes écologiques), Coma, Harvest et Chromosome 6 (corruption et trafic d'organes), The Surgeon, The Apprentice et A Deadly Practice (perversité et milieu hospitalier), Life Support (pègre et trafic génétique) et enfin, dans Vector et The Patient (bio-terrorisme).

61 Il serait d'ailleurs intéressant de savoir quelles influences littéraires, en particulier quels thrillers, ont pu laisser leur empreinte sur l'œuvre des auteurs de romans à suspense à dominante médicale. La question a été soumise à quatre écrivains ; une synthèse de leurs réponses respectives est présentée dans la section suivante.

Du point de vue narratif, le récit débute généralement par un prologue et se termine par un épilogue ${ }^{8}$, frontières implicites entre le paratexte et le texte. Ils constituent un cadre narratif et chronologique formel.

63 La fonction première du prologue est d'aiguiser l'appétit du lecteur et de lui offrir un condensé des événements à venir. Le prologue rend le futur tangible, comme c'est le cas dans Vital Signs où il annonce le drame qui va se nouer en mêlant vocabulaire spécialisé et métaphores :

The infecting bacteria came in a swift gush, as if flushed from a sewer. In an instant, several million slender, rod-shaped micro-organisms filled the lumen of the fallopian tubes. Most were grouped in small, tight clumps. They settled against the velvety convolutions of the mucosa, nestling in the warm, fertile valleys, absorbing the abundant nutrients and expelling their own foul excretions [...]. Yet, there was more to this episode than Rebecca could have known. The bacteria had started a chain of events that went beyond the immunological. The consequences were destined to come back to haunt her, to rob her of her happiness, and eventually, indirectly, to kill her. (Cook $1992: 1-3$ )

L'épilogue correspond souvent à la conclusion formelle du roman, mais parfois, il ouvre également des perspectives narratives et éditoriales ${ }^{9}$ pour l'avenir.

Quant au procédé classique des indications chronologiques (jour, date, année et heure précise) qui servent d'en-tête aux chapitres de nombreux medical thrillers, il constitue un emprunt au genre du roman à intrigue ou à suspense. La présence de ces références détaillées permet de fournir un cadre chronologique, condition indispensable au développement $\mathrm{du}$ suspense. C'est un procédé narratif utilisé initialement par M. Crichton dans A Case of Need et repris assez systématiquement par R. Cook. Dans Coma, l'auteur va même jusqu'à donner la date et l'heure à la minute près. Ces indications temporelles font office d'en-tête de chapitre.

Le milieu médical même dans lequel évoluent les personnages conditionne la narration dans les romans à suspense à dominante médicale. Qu'il s'agisse de l'hôpital, de la médecine urgentiste ou généraliste, les lieux professionnels les plus souvent représentés sont le service des urgences (Unnatural Causes de L.R. Robinson; Critical Judgement de M. Palmer; The Surgeon de T.Gerritsen), le service des soins intensifs (The Patient de M. Palmer) et le service médico-légal (Chromosome 6 de R. Cook ; Fatal de M. Palmer).

67 Ces lieux médicaux éminemment spécialisés, par nature ou intrinsèquement évocateurs de danger ou de mort, sont propices à la construction progressive du suspense. Les scènes chaotiques qui s'y déroulent sont décrites par des auteurs qui connaissent bien les rouages de ces différents services ${ }^{10}$. 


$$
\text { personnages principaux est particulièrement claire dans cet extrait de Unnatural Causes : }
$$

Well, it's a long story. My parents had always steered me toward a career in medicine. My mother's the director of nursing at a large hospital center in New Jersey, and my father's a pediatric neurologist there. To make them happy, I took a double major in English and biology. (Robinson $2001: 53$ )

71 L'influence de la formation professionnelle initiale des auteurs est telle que, même dans un contexte narratif non médical, le choix des figures de rhétorique ramène le lecteur à la réalité médicale. Dans The Apprentice, l'agent du FBI Dean, venu seconder le détective Rizzoli sur la scène du crime, est comparé à un chirurgien : " She watched as Dean gracefully balanced on one leg to pull on a shoe cover. He thrust his long hands into gloves, like a surgeon preparing to operate » (Gerritsen $2003: 161$ ).

72 Cette scène entre les personnages principaux de Invasion donne lieu à une allusion médicale alors que l'atmosphère générale est proche de celle des romans de sciencefiction :

"You're going to be okay," Beau said.

Cassy shuddered. It was the cliché that was always told to the patient no matter

what the prospective prognosis. (Cook 1997 : 269)

73 Toutefois, les scènes purement médicales sont minoritaires dans certains medical thrillers récents qui privilégient les rebondissements de l'intrigue. Dans ces conditions, il est préférable de parler de romans à suspense à influence médicale.

74 Ainsi, R. Cook met-il en avant l'action dans Chromosome 6 et la science-fiction dans Invasion . Les scènes médicales de The Apprentice représentent moins de dix pour cent de la trame narrative du roman.

\subsection{Un tissu linguistique à dominante professionnelle}

Il importe désormais de vérifier si le lien étroit entre substrat professionnel et narration a son pendant dans la trame linguistique des medical thrillers. La pertinence de l'étude des caractéristiques discursives et linguistiques propres à chaque communauté professionnelle a été soulignée par E. Benveniste :

On pourrait vérifier ainsi sur un modèle réduit le rôle de la langue à l'intérieur de la société en tant que cette langue est l'expression de certains groupes professionnels spécialisés, pour qui leur univers est l'univers par excellence. (1974 : 459-460)

Le style télégraphique émaillé d'abréviations et de termes techniques présent dans la plupart des romans à suspense à dominante médicale reflète le discours à la fois concis et précis des urgentistes. Ce type de discours spécialisé peut désorienter le lecteur non averti, mais il établit un cadre professionnel crédible. 

au service de la narration afin de faire partager au lecteur l'urgence dramatique de la situation :

'Thank God, you're back,' Ramsey cried. 'Incoming. MVA - we don't know anything except she's burned and there were fatalities at the scene.' ETA two minutes.' [...] By now the sirens were whooping down Amsterdam Avenue. We lined up by the ambulance bay door. The beep-beep-beep now of the ambulance backing into the bay. We threw open the doors and ran out onto the dock. The paramedics were already rolling.

'Unknown female, Asian, approximately mid-thirties, she was the driver of the vehicle, three fatalities,' one of the medics announced as he propelled the gurney into the emergency room. 'BP one-eighty over ninety.' (Robinson 2001 : 231-232)

Le vocabulaire spécialisé (MVA, ETA, BP one-eighty over ninety.... ${ }^{11}$, contribue à l'effet de réel. Ce type de discours génère une certaine unité situationnelle et communicationnelle. Ceci fait écho à la théorie de J.M. Swales sur les codes du discours et de la communication au sein des communautés socio-professionnelles: "Established members of discourse communities employ genres to realize communicatively the goals of their communities» (1990: 52).

Dans The Patient, le lecteur n'a pas de difficulté de compréhension particulière. Le seul terme technique, "the temporalis muscle », est explicité par le narrateur et l'image de « the question-mark incision » est transparente :

Working with practiced quickness, Jessie made a question-mark incision just over Sara's left ear, then worked the scalp away and began freeing the temporalis muscle - the broad muscle that closes the jaw. All the while, she was concentrated on locating the upper branch of the facial nerve and the vessels arising off the temporary artery. Cutting through any of those structures would have permanent consequences. (Palmer 2001: 56)

Dans The Surgeon, le discours se complexifie quelque peu («bowel sounds, paracentesis, a central line, Ringer's lactate »), mais le lecteur est à même de comprendre l'essentiel :

Ron Littman, the senior surgical resident, gave her a rapidfire report. "John Doe Pedestrian, hit-and-run. Rolled into the E.R. unconscious. Pupils are equal and reactive, lungs are clear, but the abdomen's distended. No bowel sounds. BP's down to sixty over zip. I did a paracentesis. He's got blood in his belly. We've got a central line in, Ringer's lactate wide open, but we can't keep his pressure up." (Gerritsen $2002: 19-20)$

81 En revanche, la compréhension par le profane de ces quelques lignes extraites de Shock est soumise à l'utilisation d'un dictionnaire médical. Le seul effort de vulgarisation fait par l'auteur est l'utilisation de l'expression courante « belly button» pour éclairer le sens du terme strictement médical « umbilicus » :

Paul's first goal was to introduce the trocar of the insufflation unit to fill the patient's abdominal cavity with gas. It was the creation of a gap-filled space that made the laparoscopic surgery possible. Sheila helped by grabbing two bites of skin alongside Kristin's belly button with towel clips and pulling up on the relaxed abdominal wall. Meanwhile, Paul made a small incision at the umbilicus and then proceeded to push in the foot-long Veress insufflation needle. (Cook $2002: 8$ )

Dans un souci de clarification, il n'est pas rare que le narrateur (ou un personnage) intervienne afin d'expliquer une abréviation ou un terme technique. Comme le montrent ces quelques reformulations malicieuses tirées de Unnatural Causes, la réalité parfois sèche du discours médical spécialisé doit s'accommoder de quelques aménagements nécessaires à la compréhension : 
Vittorio was not what you would call a compliant patient... He skied the most expert, black-double-diamond slopes; twice he'd sustained tib-fib fractures (both bones in the lower legs). Sailing to him meant offshore in vessels that had no business leaving sheltered bays. So far he'd been hospitalized on one occasion for hypothermia (freezing your ass), and on another for concussion (the boom on the boat swung around and hit him in the head, knocking him overboard). (Robinson $2001: 43)$

Soucieux d'éclairer le lecteur non initié, F.G. Slaughter a recours au même procédé explicatif. Non content de reformuler un terme pourtant largement connu («cartilage»), il propose une alternative plus familière, «breastbone », au mot « sternum » :

What would have been gibberish to the uninitiated was perfectly understandable to Mark. Tietze's syndrome was a very painful condition in which the cartilage gristle to the lay person - attaching the end of a rib to the breastbone or sternum was partially ruptured, causing intense chest pain that could easily be mistaken for angina. "Floppy mitral" described an anatomical abnormality found in perhaps twenty percent of the general population without causing any symptoms - except in a few. (Slaughter1983:47)

Les auteurs vont parfois jusqu'à insérer des documents (pseudo) authentiques comme une fiche de suivi de patient dans A Case of Need (Crichton 1969 : 9) ou des graphiques dans The Terminal Man (Crichton 2002 : 127-128). Cette insertion dans le corps du texte d'éléments spécialisés renforce la prégnance du réel médical dans le roman. En voici une illustration extraite de Flashback:

Brief operative note (full note dictated): ... Four-inch gash over T-10, 11, and 12 debrided ... hemostasis attained ... wound explored. ... Jagged five centimeter by three centimeter piece of rusty metal removed without difficulty ... dura appears intact; ... No collection of blood noted. ... Wound irrigated copiously, and then closed with drain in place. ... Patient sent to recovery room in stable condition, still unable to move either lower extremity. ... Tetanus and antibiotic prophylaxis initiated. ... Preoperative impression: foreign body, low midback; postoperative impression: same, plus paraplegia - etiology uncertain, possibly secondary to spinal cord disruption or circulatory embarrassment. (Palmer 1995 : 316)

$\mathrm{Au}$ vu de ces divers exemples, il est possible de dire que le discours type de la plupart des medical thrillers est à dominante médicale, même si des aménagements ponctuels (reformulations, définitions...) sont apportés pour faciliter la compréhension. Toutefois, il convient d'ajouter que le discours médical est proportionnel au substrat professionnel contenu dans la trame narrative ${ }^{12}$.

D'un point de vue purement linguistique, il serait intéressant de vérifier si les théories de plusieurs chercheurs sur le discours spécialisé dans un contexte purement professionnel sont applicables à la FASP médicale. C. Candlin a étudié différents modes de communication dans le cadre de consultations médicales formelles. Malheureusement, dans la plupart des medical thrillers, ce type de communication n'est pas dominant car les patients se retrouvent fréquemment dans des situations (service des urgences, soins intensifs...) où ils ne sont pas ou peu en mesure de communiquer. T. Varttala a observé les stratégies de communication mises en œuvre dans les articles médicaux spécialisés. Son analyse de la stratégie du hedging pourrait fort bien être appliquée au domaine de la FASP médicale :

On the one hand, hedging may take place when the precision level of exact references or numerical expressions is lowered to meet the interests of a nonspecialist audience. On the other hand, hedging may be applied in a seemingly opposite manner so as to make sure that the readership draws the desired 
conclusions from the information presented. In both cases, hedging takes place when the specialist author aims at adjusting the information to the assumed earlier knowledge of a less educated audience. (Varttala 1999 : 191-192) interpersonnelle entre médecins et/ ou chirurgiens, courantes dans les romans à suspense à dominante médicale. De telles études dépassent le cadre de cet article, mais elles pourraient faire l'objet d'un travail de recherche ultérieur.

\section{Milieux professionnels et romans à suspense à dominante médicale : le point de vue de quatre écrivains américains}

\subsection{Nature du questionnaire}

Après avoir défini la FASP médicale en tant que sous-groupe générique de la fiction à substrat professionnel ${ }^{13}$, il nous a paru utile de comparer le résultat de notre étude avec le vécu d'auteurs de romans à suspense à dominante médicale. Afin d'étudier au mieux la perméabilité entre le milieu professionnel initial - celui de la médecine - et l'œuvre de fiction, nous avons soumis en septembre 2003 un questionnaire ciblé à quatre auteurs de medical thrillers : R. Cook, M. Palmer, L.R. Robinson et T. Gerritsen.

À l'exception de R. Cook, notre questionnaire a été soumis aux quatre écrivains sous forme électronique en utilisant leurs sites personnels ou professionnels ${ }^{14}$. Il comporte huit questions. Nous présentons ici les questions posées et une synthèse des éléments les plus pertinents de leurs réponses ${ }^{15}$.

\subsection{De l'autre côté du miroir : synthèse des réponses}

4.2.1. At this stage, do you consider yourself as a doctor who writes or as a writer who happens (or happened) to be a doctor?

Il semble que l'empreinte du milieu professionnel initial reste forte chez les auteurs de la première génération. Il convient de noter que les commentaires de L.R. Robinson sont parfois ambigus car, bien qu'étant auxiliaire médicale dans un service des urgences d'un hôpital new-yorkais, elle ne se considère pas comme un médecin à part entière.

91 4.2.2. What motivations caused you to switch from medicine to fiction?

R. Cook prétend avoir été motivé par un souci de vulgarisation. T. Gerritsen affirme clairement avoir délaissé définitivement la pratique de la médecine pour l'écriture par vocation littéraire. En ce qui concerne les raisons qui ont poussé ces auteurs à privilégier l'écriture, elles sont également d'ordre familial et pratique (M. Palmer et T. Gerritsen).

4.2.3. How familiar were you with the thriller genre when you started writing?

À l'exception de T. Gerritsen, les auteurs n'avaient pas une connaissance approfondie du genre du thriller quand ils se sont aventurés sur le terrain de l'écriture. Toutefois, de nombreux écrivains spécialisés dans le roman policier ou à énigme - au rang desquels M. Connelly, P.D. James, mais aussi S. King - les ont influencés. Il est intéressant de noter l'émulation précoce entre M. Palmer et R. Cook et l'influence de M. Crichton et R. Cook sur les écrivains de la deuxième génération de la FASP médicale. 
93 4.2.4. In your view, what are the narrative techniques that best fit the atmosphere and the purposes of medical thrillers?

Le contenu narratif des medical thrillers semble étroitement lié au milieu professionnel initial. Tous les auteurs insistent sur le fait que l'association d'un certain réalisme médical reposant sur leur expérience personnelle et d'une intrigue fondée sur le mystère constitue la pierre angulaire du roman à suspense à dominante médicale. R. Cook et T. Gerritsen indiquent que l'hôpital et son cortège de peurs se prêtent bien à la rédaction des romans à suspense à dominante médicale. En outre, T. Gerritsen affirme qu'une adaptation du discours spécialisé est indispensable.

4.2.5. Do you work or exchange views with other writers of medical thrillers?

Les différents auteurs ont tendance à travailler en solitaire, ce qui ne plaide pas en faveur de l'existence d'une «École de la Nouvelle-Angleterre » formelle. En effet, leur seul travail collaboratif notoire est celui des ateliers d'écriture co-organisés dans le Massachusetts par T. Gerritsen et M. Palmer.

95 4.2.6. What do you think of the assumption that there might be a «New England School" in the field of medical fiction?

Même si R. Cook se pose en père fondateur, notre hypothèse d'une « École de la NouvelleAngleterre " fondée sur la collaboration entre les principaux auteurs ne se vérifie pas. Toutefois, le rôle prépondérant et fédérateur de l'environnement médical exceptionnel de la Nouvelle-Angleterre dans le développement du roman à suspense à dominante médicale est reconnu de façon explicite.

96 4.2.7. Do you personally believe in the pedagogical value of medical thrillers?

Les auteurs pensent qu'ils font œuvre de vulgarisation, voire de pédagogie, en raison des sujets scientifiques ou d'actualité abordés dans leurs romans. R. Cook et M. Palmer soulignent la portée éthique, voire politique, des sujets qu'ils traitent.

97 4.2.8. In what pedagogical context(s) are your novels used in the United States?

À l'exception des romans de L.R. Robinson, leurs medical thrillers sont utilisés à des fins pédagogiques dans le secondaire et le supérieur aux États-Unis. Après un contact électronique complémentaire avec T.Gerritsen, il nous est possible de dire qu'un enseignant d'un lycée du Maine (The Maine School of Science and Mathematics), John Corrigan, utilise régulièrement les romans de T. Gerritsen - en particulier Gravity - dans ses cours de littérature. Bill Pomidor, qui enseigne dans une faculté de médecine de l'Ohio, s'en sert également comme œuvre de référence pour son cours Medical School Class in Fiction. Quant au professeur Gary Goshgarian (Department of English, Northeastern University), il propose à ses étudiants l'étude de Harvest dans son cours intitulé Modern Bestsellers. Il est intéressant de noter que G. Goshgarian écrit également des medical thrillers (cf. Gray Matter, Elixir) sous le nom de plume de Gary Braver.

\section{Conclusion}

L'étude des principaux romans à suspense à dominante médicale permet d'affirmer que les milieux professionnels de la santé peuvent se reconnaître dans le substrat professionnel qui les caractérise. L'intégration, à partir du genre littéraire classique du thriller, d'éléments linguistiques et socio-professionnels propres au domaine médical donne lieu à un faisceau de caractéristiques communes fortes, emblématiques du sousgroupe générique de la FASP médicale. 
L'extrait suivant du paratexte éditorial de Unnatural Causes résume bien ces éléments fédérateurs: "Interesting and credible characters [...] an intelligent and gutsy heroine [...] realistic medical emergencies » (commentaire d'un journaliste du Daily Telegraph).

Ce substrat professionnel crédible, car proposé par des auteurs issus de la communauté médicale, est associé à une intrigue haletante fréquemment liée à un contexte médical et à un discours spécialisé souvent adapté au grand public.

La cohérence de la FASP médicale est assurée par l'expérience professionnelle initiale des auteurs, leur désir de vulgarisation et le rôle fédérateur de l'environnement médical propice de la Nouvelle-Angleterre. Comme le montrent les diverses utilisations pédagogiques des medical thrillers aux États-Unis, les romans à suspense à dominante médicale sont susceptibles de trouver un écho favorable auprès des étudiants et des professionnels de la santé.

Ce travail de recherche nous incite à penser que le roman à suspense à dominante médicale présente des caractéristiques suffisamment crédibles et attractives pour provoquer une envie de lecture accrue auprès des membres des professions médicales qui souhaitent progresser en anglais. Le chercheur y trouvera un terrain fertile, car bien des questions restent en suspens, voire en suspense.

\section{BIBLIOGRAPHIE}

\section{Références du corpus}

Braver, G. 2001 [2000]. Elixir. New York : Tor.

Braver, G. 2004 [2002]. Gray Matter. New York : Tor.

Carlson, P. 2001 [2000]. Final Duty. Londres : Arrow.

Clancy, T. 1988 [1984]. The Hunt for Red October. Glasgow : Fontana/Collins.

Connelly, J. 1999 [1998]. Bringing out the Dead. New York : Vintage Contemporaries.

Cook, R. 1978 [1977]. Coma. New York : Signet.

Cook, R. 2000 [1982]. Fever. New York : Berkley Books.

Cook, R. 1992 [1991]. Vital Signs. New York : Berkley Books.

Cook, R. 1997. Invasion. New York : Berkley Books.

Cook, R. 1998 [1997]. Chromosome 6. New York : Berkley Books.

Cook, R. 2000 [1999]. Vector. New York : Berkley Fiction.

Cook, R. 2002 [2001]. Shock. New York : Berkley Fiction.

Crichton, M. [Jeffery Hudson]. 1969 [1968]. A Case of Need. New York : Signet.

Crichton, M. 2002 [1972]. The Terminal Man. New York : Avon Books.

Gerritsen, T. 1997 [1996]. Harvest. New York : Pocket Star Books. 
Gerritsen, T. 1998 [1997]. Life Support. New York : Pocket Books.

Gerritsen, T. 2002 [2001]. The Surgeon. New York : Ballantine Books.

Gerritsen, T. 2003 [2002]. The Apprentice. New York : Ballantine Books.

Goldberg, L.S. 1994. A Deadly Practice. New York : Signet.

Grisham, J. 1995. The Rainmaker. Londres : Arrow Books.

Palmer, M. 1995 [1982]. The Sisterhood. New York : Bantam Books.

Palmer, M. 1995 [1985]. Side Effects. New York : Bantam Books.

Palmer, M. 1995 [1988]. Flashback. New York : Bantam Books.

Palmer, M. 1996. Critical Judgment. New York : Bantam Books.

Palmer, M. 1998. Miracle Cure. Londres : Arrow.

Palmer, M. 1999 [1994]. Natural Causes. Londres : Arrow.

Palmer, M. 2001 [2000]. The Patient. New York : Bantam Books.

Palmer, M. 2003 [2002]. Fatal, New York : Bantam Books.

Poe, E.A. 1977 [1967]. Selected Writings. Harmondsworth : Penguin Books.

Robinson, L.R. 1999 [1988]. Blood Run. New York : Avon Books.

Robinson, L.R. 2001 [1999]. Unnatural Causes. Londres : Pan Books.

Slaughter, F.G. 1983. Doctors at Risk. Londres : Hutchinson.

\section{Sites internet des auteurs}

Site de Gerritsen, T. <http://www.tessgerritsen.com>.

Site de Palmer, M. <http://www.michaelpalmerbooks.com>.

Site de Robinson, L.R. <http://www.leahruthrobinson.com>.

\section{Références}

Benveniste, E. 1974. Problèmes de linguistique générale, $2^{\mathrm{e}}$ tome. Paris : Gallimard.

Candlin, C. 1974. « Doctor-patient communication skill ». Working Paper 1-4, University of Lancaster, Department of Linguistics and Modern English Language, Lancaster.

Charpy, J-P. 2002 [1998]. Médias, médium et modalité. Thèse de doctorat. Villeneuve d'Ascq : Presses universitaires du Septentrion.

Charpy, J-P. 2004. « Le roman à suspense à dominante médicale : typologie d'un sous-groupe générique ». In Petit M.\& S. Isani (dir.), Aspects de la fiction à substrat professionnel. Bordeaux : Collection travaux EA 2025, Université Bordeaux 2, 65-82.

Diaz-Santos, G. 2000. « Technothrillers and English for science and technology ». English for Specific Purposes 19, 221-236.

Fowler, A. 1982. An Introduction to the Theory of Genres and Modes. Cambridge, MA : Harvard University Press.

Genette, G. 1987. Seuils. Paris : Seuil.

Lane, P. 1992. La périphérie du texte. Paris : Nathan. 
Petit, M. 1999. « La fiction à substrat professionnel : une autre voie d'accès à l'anglais de spécialité ». ASp 23-26, 57-81.

Petit, M. \& S. Isani (dir.). 2004. Aspects de la fiction à substrat professionnel. Bordeaux : Collection travaux EA 2025, Université Bordeaux 2.

Sauvageot, A. 1987. Figures de la publicité, figures du monde. Paris : Presses Universitaires de France. Swales, J. M. 1990. Genre Analysis: English in Academic and Research Settings. Cambridge : Cambridge University Press.

Varttala, T. 1999. « Remarks on the communicative functions of hedging in popular scientific and specialist articles on medicine ». English for Specific Purposes 18/2, 177-200.

\section{NOTES}

1. Les légendes arthuriennes, les romans gothiques, mais surtout les nouvelles d'E.A. Poe et les romans d'A.C. Doyle comportaient déjà des éléments annonciateurs du thriller.

2. Dans un contexte plus récent, Bringing out the Dead de J. Connelly est un roman à forte influence médicale, proche de l'autobiographie, qui décrit avec talent les affres de la vie professionnelle et émotionnelle d'un ambulancier new-yorkais. Il ne s'agit toutefois pas d'un roman à suspense.

3. Après plus de vingt ans de pratique professionnelle à Cape Cod, M. Palmer est toujours très impliqué dans les services sociaux des milieux médicaux du Massachusetts auxquels il consacre plus de vingt heures par semaine.

4. L.C. Goldberg, un auteur de la côte ouest, a également exploité cette tendance " gothique » du roman à suspense à dominante médicale (cf. A Deadly Practice).

5. Chaque automne, T. Gerritsen et M. Palmer participent au séminaire «SEAK's Annual Medical Fiction Writing for Physicians ", Sea Crest Oceanfront Resort, Falmouth, Cape Cod, Mass.

6. Dans cet article (Charpy 2004), l'auteur esquisse l'historique du roman à suspense à dominante médicale et tente de préciser les principales lignes de force du genre. Il présente également les premières constatations faites à partir d'un travail pédagogique impliquant des médecins et des étudiants en médecine autour du roman de R. Cook, Fever.

7. Les titres sont généralement courts (un ou deux mots) et incisifs (cf. références du corpus).

8. Cette technique narrative est quasiment systématique chez R. Cook et usuelle chez M. Palmer.

T. Gerritsen, S.L. Goldberg et L.R. Robinson ont principalement recours à un épilogue pour conclure leurs romans.

9. À ce sujet, voir l'épilogue de The Surgeon (2001) qui annonce clairement la suite de ce roman, The Apprentice (2002).

10. L.R. Robinson travaille comme auxiliaire médicale volontaire dans le service des urgences d'un hôpital new-yorkais. M. Palmer a travaillé pendant plus de vingt ans dans le service des urgences d'un hôpital de Boston.

11. Motor Vehicle Accident, Estimated Time of Arrival, Blood Pressure.

12. Voir à ce sujet le cas de The Apprentice (cf. 3.1.), qui peut être décrit comme un roman à influence (et non plus à dominante) médicale d'un point de vue linguistique.

13. Les sources bibliographiques concernant ce sous-groupe générique sont rares. Les sites suivants peuvent toutefois apporter des informations parcellaires, mais utiles: <Erreur! Référence de lien hypertexte non valide. et <Erreur ! Référence de lien hypertexte non valide.

14. À notre connaissance, R. Cook n'a pas de site Internet officiel. Nous avons obtenu son adresse personnelle grâce à un des auteurs.

15. Les réponses des auteurs verbatim peuvent être demandées à l'auteur de l'article. 


\section{RÉSUMÉS}

Le but de cette étude est de mettre en lumière les relations entre les milieux professionnels de la médecine et la fiction à substrat professionnel (FASP) et de les analyser. Il est convenu de dire que les medical thrillers, dont les caractéristiques principales en tant que sous-groupe générique de la FASP sont présentées ici, offrent un reflet plutôt fidèle du monde de la médecine. L'auteur montre l'intégration en profondeur du discours et des pratiques de la communauté médicale dans la trame paratextuelle, narrative et linguistique des romans à suspense à dominante médicale. Afin de mieux comprendre les relations entre l'environnement professionnel et l'œuvre de fiction, l'auteur présente les résultats d'un questionnaire soumis à quatre médecins devenus écrivains : R. Cook, M. Palmer, L.R. Robinson et T. Gerritsen.

The purpose of this study is to highlight and analyse the relationship between the medical profession and professionally-based fiction (PBF). It is generally agreed that medical thrillers, whose specific features as a sub-group of the PBF genre are presented in this paper, are a rather accurate reflection of the world of medicine. The author shows that, beneath the surface, medical PBF integrates the specific nature of the discourse and practices of the medical community into the paratextual, narrative and linguistic fabric of medical suspense novels. In order to better understand the links between professional background and fiction, the author presents the answers to a questionnaire submitted to four doctors-turned-novelists: R. Cook, M. Palmer, L.R. Robinson and T. Gerritsen.

INDEX

Mots-clés : FASP, médecine, milieu professionnel, roman à suspense à dominante médicale Keywords : medical thriller, medicine, professional background, professionally-based fiction

\section{AUTEUR}

\section{JEAN-PIERRE CHARPY}

Jean-Pierre Charpy est professeur agrégé d'anglais hors-classe à la Faculté de médecine de Dijon. Il a soutenu une thèse intitulée « Médias, médium et modalité » en 1998 à l'Université de Bourgogne. Il est le co-auteur du CD-ROM « La communication orale scientifique en anglais » (Edulang : 2003). Ses domaines de recherche portent sur la médiologie, l'utilisation des TICE et la fiction à substrat professionnel médicale. jean-pierre.charpy@u-bourgogne.fr 